\title{
Traditional open-bay versus single-family room neonatal intensive care unit: a comparison of selected nutrition outcomes
}

This article was published in the following Dove Press journal:

Research and Reports in Neonatology

29 March 20II

Number of times this article has been viewed

\author{
Christina Erickson' \\ Kendra Kattelmann' \\ Jessica Remington' \\ Cuirong Ren ${ }^{2}$ \\ Carol C Helseth ${ }^{3}$ \\ Dennis C Stevens ${ }^{3}$ \\ 'Department of Health and \\ Nutritional Sciences, ${ }^{2}$ Department \\ of Plant Science, South Dakota \\ State University, Brookings, SD, USA \\ ${ }^{3}$ Sanford Children's Hospital, \\ Sioux Falls, SD, USA
}

Background: In contrast to the traditional open-bay-type design of the neonatal intensive care unit (tNICU), infants in developmentally appropriate NICU (dNICU) are housed in individual rooms with greater control of light and noise. Previous reports have documented positive influence of the dNICU in cardiorespiratory status, physiologic stability, and weight gain of the infants. The objective of this study was to explore selected nutrition outcomes of infants in the dNICU versus tNICU.

Method: A prospective cohort study was conducted on infants with birth weight of $1500 \mathrm{~g}$ or less cared for in dNICU $(n=42)$ or tNICU $(n=31)$. Differences between days to reach full parenteral nutrition, full enteral nutrition, or full bottling were determined using analysis of covariance controlling for gestational age, birth weight, and clinical risk index for babies (CRIB) acuity score.

Results: There were no differences between the two groups in days to reach full parenteral and bottle feeding. The infants in the dNICU took fewer days to reach full enteral nutrition (20.8 days, 95\% confidence intervals [CI]: 17, 24.6 (dNICU) vs 23.3 days, 95\% CI: 17.1, 29.6 (tNICU), $P=0.04$ ) than those in the tNICU.

Conclusions: Although the two groups of infants only differed in the days to reach full enteral feeding, it is important to remember that the lack of difference may be clinically significant. Clinically, the infants in the dNICU were younger (gestational age) and sicker (CRIB acuity score) than the infants in the tNICU. Consequently, the results of this study support the change to dNICU, as the private room model provides a supportive environment for growth as evidenced by similar nutritional outcome measures. More research is needed to determine the effect of the dNICU on nutrition outcomes.

Keywords: NICU, neonate, infant, feeding

\section{Introduction}

The neonatal intensive care unit (NICU) is vital to the survival of preterm infants. However, traditional ward-style NICU (tNICU) may involve environmental stress to the neonate and are potentially detrimental for infants lacking mature organ systems and the ability to adapt to abrupt changes. ${ }^{1-4}$ Consequently, NICUs are being redesigned into more developmentally appropriate environments with single rooms, controlled light and noise, clustering medical care to promote rest, and a family-centered approach to infant care. Single-family room-style NICUs (developmentally appropriate NICU [dNICU]) aim to reduce infant stress, implement strategies to manage environmental challenges, and individualize the plan of care to meet the special needs of the preterm infant; however, the implemented strategies for the NICU vary from study to study. ${ }^{2,5-8}$
Correspondence: Kendra Kattelmann Department of Health and Nutritional Sciences, South Dakota State University, Brookings, SD 57007, USA

$\mathrm{Tel}+\mathrm{I} 6056884045$

Fax +l 6056885603

Email kendra.kattelmann@sdstate.edu 
The goal of developmental care is to support the neonate in a stable environment, thus conserving energy for appropriate growth and development. ${ }^{2}$ Single-family room-style environments are more developmentally appropriate and are becoming more prevalent as research emerges in regard to the potential benefits to the infant and the family. Preterm infant outcomes in the single-family room style are typically improved in regard to neurodevelopment and growth compared to the open-bay open-ward (traditional) NICU. ${ }^{2-4,8,9}$ However, the environment and interventions vary significantly between studies, so it is difficult to compare outcomes of one specific variation in design.

Aucott et $\mathrm{al}^{5}$ reviewed the effect of many variables of the NICU on neonatal outcomes. The variables included changes in NICU design, positioning and handling of infants, nursing care plans, nursing routines, feeding methods, parental involvement, and the implementation of Neonatal Individualized Developmental Care and Assessment Program (NIDCAP). NIDCAP includes extensive staff training in assessing infants and determining individualized care based on the infant's needs. ${ }^{5,10}$ The authors reported improved outcomes in many studies from the initiation of NIDCAP, the NICU environment, changes in positioning and handling protocols, nonnutritive suckling, family involvement, and breastfeeding. The NICU environment, including decreased sound and light at night and cycled light, was beneficial in many studies to NICU outcomes. ${ }^{5}$

The environment of the NICU may influence infant outcomes. Many studies address the amount of light, noise, and stress on an infant while in the NICU. Blackburn and Patteson ${ }^{11}$ studied the effect of cycled light on the cardiorespiratory system in preterm infants and reported decreased activity and heart rate during the low light levels compared to infants exposed to continuous light. This may be indicative of the infant being more organized in his or her sleep patterns and having less overall stress. Cycled light was also used in a study by Brandon et al, ${ }^{6}$ which compared infants with cycled light intervals to those with continuous bright light. The authors reported short-term advantages such as weight gain to cycled light protocols. Brown ${ }^{7}$ studied the effect of noise on preterm infants by reviewing many articles. The authors concluded that lower levels of noise in the NICU may improve physiologic stability of preterm infants and long-term outcomes. The design of the NICU greatly influences the preterm infants' exposure to environmental noise, light, and stress. Stevens et $\mathrm{al}^{12}$ reported significantly less sound and noise in the NICU after conversion from a conventional open-bay layout to a single-patient room layout.
Although there are many benefits to a single-room layout in the NICU, there are some challenges to the design. Carlson et $\mathrm{al}^{3}$ reported that although families are able to be a part of the team and involved with the care of the preterm infant, there are some challenges in staff satisfaction. The authors reported that overcoming the resistance to change was the most challenging aspect of the conversion to single-family rooms. Staff may have fears of not being able to monitor many babies at one time as well as the distance from the nurse to the infant. However, Stevens et $\mathrm{al}^{12}$ reported that staff views on patient care, job quality, health and safety, and security in the NICU were all greater with a single-room design than the open-room concept.

The Boekelheide NICU at Sanford Children's Hospital was converted into a single-family room-style environment in 2006 and was designed using the Recommended Design Standards for Advanced Neonatal Care. ${ }^{13}$ The single-infant rooms allow parents to room with the neonates. The Boekelheide NICU utilizes giraffe beds (GE Healthcare, Laurel, MD), which can control temperature and humidity and block light and sound. The unit also uses indirect lighting and noise reduction strategies in order to provide an environment that promotes growth and development. This study was designed prior to the conversion to compare outcomes from tNICU to dNICU. Development of the Boekelheide NICU to a single-patient room and preliminary outcomes on effects of noise reduction were previously reported by Stevens et al. ${ }^{12,14}$ The objective of this study was to explore selected nutritional outcomes before and after the change from the tNICU environment to the dNICU environment at Sanford Children's Hospital.

\section{Methods \\ Overall design}

A prospective cohort study was conducted at the Boekelheide NICU of Sanford Children's Hospital. This study was designed before the move to the single-family room dNICU to compare the outcomes from an open-bay ward tNICU to a dNICU. Two time periods were selected to compare the outcomes. Charts from November 28, 2005, to May 28, 2006, were designated as the tNICU group, and charts from November 28, 2006, to May 28, 2007, (after the move to the developmentally appropriate single-family room Boekelheide NICU) were designated as the dNICU group. Only surviving infants were used for the study. Infants weighing more than $1500 \mathrm{~g}$ at birth were excluded from the study due to an assumed decrease in need for intensive interventions and a shorter length of stay and the likelihood of having fewer complications. Infants with genetic syndromes and major 
surgery, were also excluded due to increased complications and the likelihood of higher calorie needs.

Sanford's NICU had a core of developmental specialists in both units. The core included a registered dietitian who is part of the multidisciplinary nutrition team along with other health care professionals in the fields of speech and physical therapy, nursing, social work, case management, and pharmacy and PhD-level developmental therapist. The team discusses issues, such as growth, lab values, progress, assessments, and recommendations, and has been in place since 2003. This nutrition support team used the same nutrition practice guidelines for human milk fortification, supplementation of protein, and procedures for monitoring growth and laboratory values for both time periods of this study. No major staff changes occurred between the two time periods, and the same standardized protocols and procedures were used during both periods.

\section{Data collection}

The following information was collected: length of stay in days, birth weight, discharge weight, postnatal weight loss, days to reach full parenteral nutrition, days to reach full enteral nutrition, and days to reach full bottling/nippling. Average weight gain per day was calculated by dividing the difference between discharge and birth weight by the length of stay in days. Average weight gain per day per kilogram of body weight was calculated by dividing the average weight gain per day by the kilograms of birth weight. Days to reach the nutrition-related outcomes were determined based on the first time each of the parameters was met. Full parenteral nutrition was defined as $70 \mathrm{kcal} / \mathrm{kg} /$ day, full enteral nutrition as $100 \mathrm{kcal} / \mathrm{kg} /$ day, and full bottling/nippling as $100 \mathrm{kcal} / \mathrm{kg} /$ day orally.

The following factors that could influence measured nutritional outcomes were collected for comparison between groups, and if significantly different, the variable was included as covariates in the analysis: gestational age at birth, inborn or outborn status, Apgars at 1 and $5 \mathrm{~min}$, clinical risk index for babies (CRIB) $)^{15}$ acuity score, maximum acuity score, days on oxygen support, days on continuous positive airway pressure (CPAP), days on mechanical ventilation, days on oscillator, incidence of discharge on respiratory support, and incidence of necrotizing enterocolitis and intrauterine growth retardation. All information was gathered by trained study personnel through queries in Sanford Health's Neo-data (NICU Patient Data System, Isoprime Corp, Chicago, IL) database and from paper and electronic charts.
Approval for the study was obtained through the Human Subjects Institutional Review Boards of Sanford Health Institutional Review Board and South Dakota State University. Electronic and paper charts were viewed in a private room at Sanford Health and were kept in a locked room or on a locked computer when not being viewed in order to ensure confidentiality. All patient identifiers were removed when extracting information.

\section{Statistical analysis}

Statistical analyses were performed using the SAS System (Version 9.2; SAS Institute Inc, Cary, NC). Differences between independent variables were determined using the Student's $t$-test, Fisher's exact test, and Kruskal-Wallis test for nonparametric methods based on Wilcoxon scores and Cochran-Mantel-Haenszel. Due to variability in birth weight within groups, differences in dependent variables (outcome variables) were determined using the analysis of covariance controlling for birth weight, gestational age, and CRIB acuity score. The significance criterion was defined as $P<0.05$.

\section{Results Infant statistics}

There were a total of 31 eligible charts from the tNICU and 42 eligible charts from the dNICU that met the inclusion criteria established for this study. There were significant differences between groups for gestational age between the tNICU and the dNICU (28.1 weeks, $95 \%$ confidence intervals [CI]: 27, 29.2 [ $\mathrm{n}=31]$ and 26.7 weeks, 95\% CI: 25.9, 29.2 ( $\mathrm{n}=42$ ), respectively, $P=0.05)$ and CRIB acuity score (2.7, 95\% CI: 1.6, 3.9 [n=31] and 5.2, 95\% CI: 3.8, $6.6[\mathrm{n}=42]$, respectively, $P=0.01)$. There were no differences between groups for birth weight, days on oxygen, days on CPAP, days on mechanical ventilation, days on oscillator, Apgar scores at 1 and $5 \mathrm{~min}$, maximum acuity score, inborn versus outborn status, instances of discharge on respiratory support, instance of necrotizing enterocolitis, and number with intrauterine growth retardation (Table 1).

\section{Outcome measures}

Significant differences were found between the tNICU and the dNICU in weight at discharge (3343 g, 95\% CI: 2479 , 4206 [ $\mathrm{n}=31]$ and $3162 \mathrm{~g}, 95 \%$ CI: 2777, 3546 [n = 42], respectively, $P=0.04)$, average weight gain per day $(24.7 \mathrm{~g}$, 95\% CI: 22.9, 26.4 [n $=31]$ and 22.5 g, 95\% CI: 21.1, 23.9 [n $=42], P=0.05)$, and days to reach full enteral nutrition (23.3 days, 95\% CI: 17.1, $29.6[\mathrm{n}=31]$ and 20.8 days, $95 \%$ CI: $17,24.6[\mathrm{n}=42]$, respectively, $P=0.04)$. There were no 
Table I Comparison of infant acuity variables

\begin{tabular}{|c|c|c|c|}
\hline Variable & $\begin{array}{l}\text { tNICU' mean } \\
(95 \% \mathrm{CI})(\mathrm{n}=3 \mathrm{I})\end{array}$ & $\begin{array}{l}\text { dNICU' mean } \\
(95 \% \mathrm{Cl})(n=42)\end{array}$ & $P$ value \\
\hline Gestational age (weeks) ${ }^{2}$ & $28.1(27.0,29.2)$ & $26.7(25.9,29.2)$ & 0.05 \\
\hline Birth weight $(g)^{2}$ & $1047(958,1137)$ & $952(865,1040)$ & 0.14 \\
\hline Days on oxygen support ${ }^{2}$ & $54.6(20.3,88.8)$ & $62.4(4 I .6,83.1)$ & 0.68 \\
\hline Days on continuous positive airway pressure ${ }^{2}$ & I4.7 (8.6, 20.8) & $18.0(12.3,23.7)$ & 0.42 \\
\hline Days on ventilator ${ }^{2}$ & $21.8(3.0,46.5)$ & $24.9(14.5,35.1)$ & 0.80 \\
\hline Days on oscillator ${ }^{2}$ & I.I $(0.3,2.5)$ & $1.0(0,1.8)$ & 0.85 \\
\hline Apgar at I $\min ^{2}$ & $5.5(4.7,6.2)$ & $5.3(4.6,6.1)$ & 0.79 \\
\hline Apgar at $5 \mathrm{~min}^{2}$ & $7.5(6.8,8.1)$ & $7.2(6.7,7.6)$ & 0.53 \\
\hline CRIB acuity score 3 & $2.7(1.6,3.9)$ & $5.2(3.8,6.6)$ & 0.01 \\
\hline Maximum acuity score ${ }^{2}$ & $4.3(4.0,4.5)$ & $4.3(4.0,4.5)$ & 0.98 \\
\hline Number born in another facility ${ }^{4}$ & 4 & 6 & 0.87 \\
\hline Number discharged on respiratory support ${ }^{4}$ & 7 & 15 & 0.22 \\
\hline Number with necrotizing enterocolitis ${ }^{4}$ & 5 & 2 & 0.11 \\
\hline Number with intrauterine growth retardation ${ }^{4}$ & 6 & 4 & 0.22 \\
\hline
\end{tabular}

Notes: 'tNICU is a group of infants from traditional open-bay neonatal intensive care unit. The dNICU is a group of infants from single-family room neonatal intensive care unit; ${ }^{2}$ Comparison between group differences determined using Student's $t$-test or Fisher's exact test; ${ }^{3}$ Comparison between group differences determined using KruskalWallis test from nonparametric methods based on Wilcoxon scores; ${ }^{4}$ Comparison between group differences determined using Cochran-Mantel-Haenszel.

Abbreviations: $\mathrm{Cl}$, confidence interval; CRIB, clinical risk index for babies; tNICU, traditional neonatal intensive care unit; dNICU, developmentally appropriate neonatal intensive care unit

differences between other outcome variables, such as length of stay, days to reach full parenteral nutrition, and days to reach full bottling (Table 2).

\section{Discussion}

Preterm infants rely on specialized care in the NICU for survival. The open-bay ward-style tNICUs lack resemblance to in utero and introduce the infant to a physiologically stressful, loud, bright environment filled with invasive care. The infant, lacking mature organ systems and the ability to adapt to abrupt changes, may not grow and develop appropriately due to this stress. ${ }^{1-3,14}$ Controlling the environment in the dNICU may allow for energy conservation and consequently appropriate growth and development. ${ }^{2}$ The results of this study indicate a potential nutritional benefit from developmentally appropriate care in days to reach full enteral nutrition. Babies in the dNICU reached full enteral nutrition 2.5 days sooner than babies in the tNICU. However, in these infants, reaching full enteral nutrition earlier did not result in a greater average weight gain per day or greater discharge weight. Als et $\mathrm{al}^{9}$ reported earlier oral feeding in preterm infants in the dNICU compared to the tNICU accompanied by higher average weight gain per day with developmentally appropriate care. In this study, when the weight gain per day was normalized to infants' birth weight in kilograms, there was no difference in weight gain per day.

Table 2 Selected nutrition outcome variables of infant from tNICU versus dNICU infants

\begin{tabular}{|c|c|c|c|}
\hline Variable & $\begin{array}{l}\text { tNICU' mean } \\
(95 \% \mathrm{CI} \text { or } \pm \text { SE })(n=3 I)\end{array}$ & $\begin{array}{l}\text { dNICU' mean } \\
(95 \% \mathrm{CI} \text { or } \pm \text { SE) }(n=42)\end{array}$ & $P$ value \\
\hline Length of stay (days) ${ }^{2}$ & $90(6 I, 121)$ & $96(79,112)$ & 0.73 \\
\hline Weight at discharge $(g)^{3}$ & $3343(2479,4206)$ & $3162(2777,3546)$ & 0.04 \\
\hline Average postnatal weight loss $(g)^{2}$ & $79(57,102)$ & $91(77,105)$ & 0.36 \\
\hline Average weight gain/day $(g)^{3,4}$ & $24.7(22.9,26.4)$ & $22.5(21.1,23.9)$ & 0.05 \\
\hline $\begin{array}{l}\text { Average weight gain/day normalized to } \mathrm{kg} \\
\text { birth weight }(\mathrm{g} / \mathrm{kg} / \text { day })^{3,4}\end{array}$ & $24.7(22.1,27.4)$ & $25.4(22.8,28.1)$ & 0.30 \\
\hline Days to reach full parenteral nutrition ${ }^{3,5}$ & 4.I $(3.6,4.7)$ & $5.5(4.3,6.8)$ & 0.47 \\
\hline Days to reach full enteral nutrition ${ }^{3,5}$ & $23.3(17.1,29.6)$ & $20.8(17.0,24.6)$ & 0.04 \\
\hline Postnatal days at full bottling ${ }^{2,5}$ & $57 \pm 6$ & $67 \pm 5$ & 0.50 \\
\hline
\end{tabular}

Notes: 'tNICU is a group of infants from traditional open-bay neonatal intensive care unit. The dNICU is a group of infants from single-family room neonatal intensive care unit; ${ }^{2}$ Comparison between group differences determined using Student's $t$-test or Fisher's exact test; ${ }^{3}$ Comparison between group differences determined using analysis of covariance GLM procedure controlling for CRIB acuity, gestational age, and birth weight; ${ }^{4}$ Average weight gain/day normalized to $\mathrm{kg}$ birth weight (gm/kg/day) = average weight gain/day in g/birth weight in kg. Average weight gain/day = discharge weight - birth weight/length of stay in days; ${ }^{5}$ Days to reach outcomes based on the first time parameters were met. Full parenteral nutrition was defined as $70 \mathrm{kcal} / \mathrm{kg} /$ day, full enteral nutrition as $100 \mathrm{kcal} / \mathrm{kg} / \mathrm{day}$, and full bottling/nippling as $100 \mathrm{kcal} / \mathrm{kg} / \mathrm{day}$ orally.

Abbreviations: $\mathrm{Cl}$, confidence interval; SE, standard error; CRIB, clinical risk index for babies; tNICU, traditional neonatal intensive care unit; dNICU, developmentally appropriate neonatal intensive care unit; GLM, general linear model. 
Although many of the physiological status variables reviewed were not significantly different between the dNICU and the tNICU environments, it was important to note that the infants in the dNICU were younger (gestational age) and sicker (CRIB acuity score) than the infants in the tNICU. Infants born earlier are less mature than those born later, and so the adaptation of the immature organ systems might have been more challenging for the dNICU infant. Clinically, another consideration is the age of the infants at discharge. The infants in the tNICU were discharged at a slightly greater gestational age than those in the dNICU. The lower weight at discharge of the dNICU infant might be due to the slightly younger gestational age at discharge.

The goal of the dNICU is to reduce infant stress, implement strategies to manage environmental challenges, and individualize the plan of care to meet the special needs of the preterm infant. ${ }^{2,8}$ To meet these requirements, infants in the dNICUs are housed in private rooms, and consequently, the infants may be farther away from nursing staff. Carlson et al studied the challenges faced by staff when converting to a private-room model in NICU care and reported that one challenge nursing staff face is the lack of ability to view many infants and families at one time. ${ }^{3}$ There is common fear among the staff that the distance from a central nursing station may hinder care. Based on the results from this study, these fears are unfounded as the selected measured nutritional outcomes of the dNICU compared to the tNICU were the same or better. It appears as though the benefits of the dNICU outweigh the reasons for resistance to change.

A potential study limitation is the design. Because of the nature of the study of the investigation, it could not be randomized and blinded. The study was a prospectively designed observational study that occurred due to the opportunity to upgrade and build a new NICU. However, the study was preplanned, and due to the complexity and nature of the research design and moving to a new facility, data were collected 6 months after moving to the new facility. Furthermore, there were no major changes in staffing, and both the tNICU and the dNICU had the same core of developmental specialists in both units. The core included a registered dietitian and used the same nutrition practice guidelines. Sneve et al ${ }^{16}$ studied NICU outcomes with a registered dietitian as a part of the NICU team and reported significant improvements with a registered dietitian on staff. However, even with the same staff and protocol, improved quality of care over time may have inherently influenced the outcomes.

Additionally, the small sample size may be a limiting factor in the interpretation of the results. A larger sample size may be needed. A power calculation based on results from a similar article by Als et $\mathrm{al}^{9}$ suggests that a sample size of 42 from each group is needed.

\section{Conclusion}

This study demonstrated a benefit of dNICU care in days to reach full enteral feeding over the tNICU. However, there were no differences between other outcome variables, such as length of stay, days to reach full parenteral nutrition, and days to reach full bottling. Additionally, there is a growing body of evidence that controlling the environment in the NICU is associated with benefits to the infant and the families. . $^{1,2,10,11,14,17}$ Further research is necessary in regard to nutrition outcomes of developmentally appropriate care in the NICU.

\section{Acknowledgment}

Graduate student stipends for Christina Erickson and Jessica Remington were supported by Leadership Education in Neurodevelopmental Disabilities Program funded by Maternal and Child Health Bureau of Health Resources and Services Administration (HRSA) grant number T73MC00037.

\section{Disclosure}

The authors report no conflicts of interest in this work.

\section{Authors' contributions}

$\mathrm{CE}, \mathrm{JR}, \mathrm{CH}$, and DS have made substantial contributions to conception and design and acquisition of data. CR and $\mathrm{KK}$ made substantial contributions to analysis and interpretation of data. CE, JR, and KK have been involved in drafting the manuscript and revising it for critically important intellectual content. DS has given final approval of the version to be published. All authors have participated sufficiently in the work to take public responsibility for appropriate portions of the content. All authors contributed equally to this work.

\section{References}

1. Atun-Einy O, Scher A. Measuring developmentally appropriate practice in neonatal intensive care units. J Perinatol. 2008;28(3):218-225.

2. Byers JF. Components of developmental care and the evidence for their use in the NICU. MCN Am J Matern Child Nurs. 2003;28(3):174-180.

3. Carlson B, Walsh S, Wergin T, Schwarzkopf K, Ecklund S. Challenges in design and transition to a private room model in the neonatal intensive care unit. Adv Neonatal Care. 2006;6(5):271-280.

4. Zahr LK. Two contrasting NICU environments. MCNAm J Matern Child Nurs. 1998;23(1):28-36.

5. Aucott S, Donohue PK, Atkins E, Allen MC. Neurodevelopmental care in the NICU. Ment Retard Dev Disabil Res Rev. 2002;8(4):298-308.

6. Brandon DH, Holditch-Davis D, Belyea M. Preterm infants born at less than 31 weeks' gestation have improved growth in cycled light compared with continuous near darkness. J Pediatr. 2002;140(2):192-199. 
7. Brown G. NICU noise and the preterm infant. Neonatal Netw. 2009;28(3): 165-173.

8. McGrath JM, Conliffe-Torres S. Integrating family-centered developmental assessment and intervention into routine care in the neonatal intensive care unit. Nurs Clin North Am. 1996;31(2): 367-386.

9. Als H, Lawhon G, Duffy FH, McAnulty GB, Gibes-Grossman R, Blickman JG. Individualized developmental care for the very low-birthweight preterm infant. Medical and neurofunctional effects. JAMA. 1994;272(11):853-858.

10. McAnulty G, Duffy FH, Butler S, et al. Individualized developmental care for a large sample of very preterm infants: health, neurobehaviour and neurophysiology. Acta Paediatr. 2009;98(12): $1920-1926$.

11. Blackburn S, Patteson D. Effects of cycled light on activity state and cardiorespiratory function in preterm infants. J Perinat Neonatal Nurs. 1991;4(4):47-54.

12. Stevens DC, Helseth CC, Khan MA, Munson DP, Smith TJ. Neonatal intensive care nursery staff perceive enhanced workplace quality with the single-family room design. $J$ Perinatol. 2010;30(5):352-358.
13. Consensus Committee on Recommended Design Standards for Advanced Neonatal Care. Recommended Standards for Newborn ICU Design. February 1, 2007. Available from: http://www.nd.edu/ nicudes/ Recommended\%20Standards\%207\%20final\%20may\%2015.pdf. Retrieved October 1, 2007. Accessed January 20, 2011.

14. Stevens DC, Akram Khan M, Munson DP, Reid EJ, Helseth CC, Buggy J. The impact of architectural design upon the environmental sound and light exposure of neonates who require intensive care: an evaluation of the Boekelheide Neonatal Intensive Care Nursery. J Perinatol. 2007; 27 Suppl 2:S20-S28.

15 The International Neonatal Network. The CRIB (Clinical Risk Index for Babies) score: a tool for assessing initial neonatal risk and comparing performance of neonatal intensive care units. Lancet. 1993;342(8865):193-198.

16. Sneve J, Kattelmann K, Ren C, Stevens DC. Implementation of a multidisciplinary team that includes a registered dietitian in a neonatal intensive care unit improved nutrition outcomes. Nutr Clin Pract. 2008-2009;23(6):630-634.

17. Anderson DM. Nutritional assessment and therapeutic interventions for the preterm infant. Clin Perinatol. 2002;29(2):313-326.

\section{Publish your work in this journal}

Research and Reports in Neonatology is an international, peer-reviewed, open access journal publishing original research, reports, editorials, reviews and commentaries on neonatal health. The manuscript management system is completely online and includes a very quick and fair peer-review system. Visit http://www.dovepress.com/testimonials.php to read real quotes from published authors. 